\title{
AN INTEGRAL REPRESENTATION FOR GENERALIZED TEMPERATURES IN TWO SPACE VARIABLES
}

\author{
DEBORAH TEPPER HAIMO ${ }^{1}$
}

ABstract. An integral representation is derived for a function which satisfies the generalized heat equation in one of the space variables and the adjoint generalized heat equation in the other space variable.

In recent papers [2]-[7], series and integral representation theories were developed for generalized temperature functions. In this note, we derive an integral representation for a function which satisfies the generalized heat equation in one of the space variables and the adjoint generalized heat equation in the other space variable.

A generalized temperature function is a $C^{2}$ solution $u(x, t)$ of the generalized heat equation

$$
\Delta_{x}(u(x, t))=(\partial / \partial t) u(x, t)
$$

where $\Delta_{x} f(x)=f^{\prime \prime}(x)+(2 \nu / x) f^{\prime}(x), \nu>0$. The adjoint generalized heat equation is given by

$$
\Delta_{x} u(x, t)+(\partial / \partial t) u(x, t)=0 .
$$

The fundamental solution of the generalized heat equation is the function

$$
G(x ; t)=\int_{0}^{\infty} e^{-t u^{2}} \mathcal{J}(x u) d \mu(u)=\left(\frac{1}{2 t}\right)^{v+1 / 2} e^{-x^{2} / 4 t}
$$

where

$$
\begin{aligned}
g(z) & =2^{\nu-1 / 2} \Gamma\left(\nu+\frac{1}{2}\right) z^{1 / 2-\nu} J_{\nu-1 / 2}(z), \\
d \mu(z) & =\frac{1}{2^{\nu-1 / 2} \Gamma\left(\nu+\frac{1}{2}\right)} z^{2 \nu} d z
\end{aligned}
$$

$J_{\alpha}(z)$ being the ordinary Bessel function of order $\alpha$. If

Received by the editors December 22, 1970 and, in revised form, March 18, 1971.

AMS 1970 subject classifications. Primary 35C15; Secondary 35K05.

Key words and phrases. Generalized temperatures, integral representations, generalized heat equation, Bessel function.

${ }^{1}$ The research of this paper was supported by the National Science Foundation under grant GP 20536.

Copyright (c) 1971, American Mathematical Society 


$$
D(x, y, z)=\frac{2^{2 v-5 / 2}\left[\Gamma\left(\nu+\frac{1}{2}\right)\right]^{2}}{\Gamma(\nu) \pi^{1 / 2}}(x y z)^{1-2 \nu}[\Delta(x, y, z)]^{2 \nu-2},
$$

where $\Delta(x, y, z)$ is the area of a triangle whose sides are $x, y, z$ if there is such a triangle, and otherwise, $D(x, y, z)=0$, the associated function $f(x, y)$ corresponding to a function $f(x)$ is given by

$$
f(x, y)=\int_{0}^{\infty} f(u) D(x, y, u) d \mu(u), \quad 0<x, y<\infty .
$$

The function associated with the fundamental solution $G(x ; t)$ is

$$
G(x, y ; t)=\int_{0}^{\infty} e^{-t u^{2}} \mathcal{g}(x u) \mathcal{J}(y u) d \mu(u)=\left(\frac{1}{2 t}\right)^{v+1 / 2} e^{-\left(x^{2}+v^{2}\right) / 4 t g}\left(\frac{x y}{2 t}\right),
$$

where

$$
g(z)=2^{\nu-1 / 2} \Gamma\left(\nu+\frac{1}{2}\right) z^{1 / 2-\nu} I_{\nu-1 / 2}(z),
$$

$I_{\alpha}(z)$ being the Bessel function of imaginary argument and order $\alpha$. A bounded function $f(x)$ for which

$$
\sum_{j=1}^{n} \sum_{k=1}^{n} a_{j} \bar{a}_{k} f\left(x_{j}, x_{k}\right) \geqq 0
$$

for any $x_{1}, x_{2}, \cdots, x_{n}>0$ and arbitrary complex numbers $a_{1}, a_{2}, \cdots$, $a_{n}$ is said to be positive definite.

We establish two theorems, each a consequence of two basic lemmas which give integral representations for functions of one space variable.

LEMMA 1. A function $u(x, t)$ has the representation

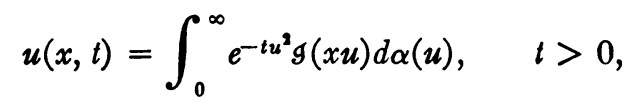

where $\alpha(u)$ is a nondecreasing function iff

(i) $\Delta_{x} u(x, t)=-(\partial / \partial t) u(x, t), t>0$,

(ii) $u(x, t) \geqq 0, t>0$.

Proof. The necessity of the conditions is immediate on noting that $g(x u) \geqq 0$ and that $\Delta_{x} \mathscr{g}(x u)=u^{2} \mathscr{g}(x u)$, with differentiation under the integral sign justifiable.

To establish sufficiency, consider the function

$$
v(x, t)=G(x ; t) u\left(\frac{x}{t}, \frac{1}{t}\right), \quad t>0 .
$$


Then it readily follows that $v(x, t)$ is a nonnegative generalized temperature function for $t>0$. Hence by $[2$, p. 49], we have

$$
v(x, t)=\int_{0}^{\infty} G(x, y ; t) d \beta(y),
$$

with $\beta(y)$ nondecreasing, or

$$
u(x, t)=\int_{0}^{\infty} e^{-y^{2} t} g(x y) d \alpha(y)
$$

where $\alpha(y)=\beta(2 y)$, and the proof is complete.

Lemma 2. A function $u(x, t)$ has the representation

$$
u(x, t)=\int_{0}^{\infty} e^{-t u^{2} g(x u) d \alpha(u), \quad t>0,}
$$

where $\alpha(u)$ is nondecreasing iff

(i) $\Delta_{x} u(x, t)=(\partial / \partial t) u(x, t), t>0$,

(ii) $u(z, t)$ is analytic for each $t>0$ and $|\operatorname{Re} z|<R$,

(iii) $u(i x, t) \geqq 0, t>0$.

Proof. The necessity of the conditions is immediate with the analyticity of $u(z, t)$ a consequence of Theorem 5.3 of [2].

Conversely, since $u(i y, t) \geqq 0$ and $u(i y, t)$ is a solution of the adjoint generalized heat equation for $t>0$, the result follows by the preceding lemma.

Combining these two results, we have the following.

ThEOREM 3. A function $u(x, y, t)$ has the representation

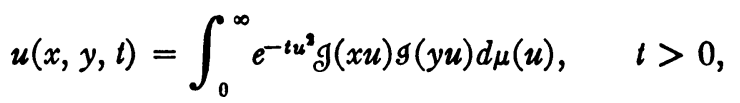

with $\alpha(u)$ nondecreasing iff

(i) $\Delta_{x} u(x, y, t)=-\Delta_{y}(x, y, t)=(\partial / \partial t) u(x, y, t)$,

(ii) for $y \geqq 0, t>0, u(z, y, t)$ is analytic for $|\operatorname{Re} z|<R$,

(iii) for each $x \geqq 0, y \geqq 0, t>0, u(i x, y, t) \geqq 0$.

Proof. The necessity of the conditions are readily verified.

Conversely, an appeal to Lemma 1 , yields, for fixed $x$,

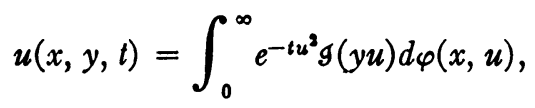

where, for each $x, \varphi(x, u)$ is nondecreasing. Hence 


$$
u(x, 0, t)=\int_{0}^{\infty} e^{-t u^{2}} d \varphi(x, u) .
$$

But, $u(x, 0, t)$ satisfies the conditions of Lemma 2 so that

$$
u(x, 0, t)=\int_{0}^{\infty} e^{-t u^{2}} g(x u) d \alpha(u)
$$

with $\alpha(u)$ nondecreasing. Consequently,

$$
\int_{0}^{\infty} e^{-t u^{2}}[\mathcal{J}(x u) d \alpha(u)-d \varphi(x, u)]=0,
$$

and since the left-hand side is a Laplace transform, it follows by uniqueness, that, for each fixed $x$,

$$
g(x u) d \alpha(u)=d \varphi(x, u),
$$

yielding the desired representation for $u(x, y, t)$.

An example illustrating the theorem is given by

$$
\left(\frac{1}{2 t}\right)^{\nu+1 / 2} e^{\left(-x^{2}+y^{2}\right) / 4 t} \mathfrak{g}\left(\frac{x y}{2 t}\right)=\int_{0}^{\infty} e^{-t u^{2}} g(x u) g(y u) d \mu(u) .
$$

We establish criteria for a similar representation, but with $\alpha(u)$ a nondecreasing bounded function, again, by first proving two basic lemmas.

LEMMA 4. A necessary and sufficient condition that

$$
u(x, t)=\int_{0}^{\infty} e^{-t u^{2}} g(x u) d \alpha(u), \quad t>0
$$

with $\alpha(u)$ nondecreasing and bounded, is that

(i) $\Delta_{x} u(x, t)=(\partial / \partial t) u(x, t), t>0$,

(ii) $u(x, t)>-M$ for some $M>0, t>0$,

(iii) $u(x, 0+)$ exists and is positive definite.

Proof. If the integral representation holds, then (i) is immediate; (ii) follows from the fact that

and (iii) from [1].

$$
|u(x, t)| \leqq \int_{0}^{\infty} d \alpha(u)<\infty, \quad t>0 ;
$$

Conversely, we note that by (i) and (ii), $u(x, t)+M$ is a positive generalized temperature function, and hence, by Corollary 8.6 of [2], 


$$
u(x, t)=\int_{0}^{\infty} G(x, y ; t) u(y, 0+) d \mu(y), \quad t>0 .
$$

But by [1], (iii) implies that

$$
u(x, 0+)=\int_{0}^{\infty} g(x u) d \alpha(u),
$$

with $\alpha(u)$ nondecreasing and bounded. Hence

$$
\begin{aligned}
u(x, t) & =\int_{0}^{\infty} G(x, y ; t) d \mu(y) \int_{0}^{\infty} \delta(y u) d \alpha(u) \\
& =\int_{0}^{\infty} d \alpha(u) \int_{0}^{\infty} \mathcal{J}(y u) G(x, y ; t) d \mu(y) \\
& =\int_{0}^{\infty} e^{-t u^{2}} \mathcal{g}(u x) d \alpha(u),
\end{aligned}
$$

the interchange in order of integration being justifiable by Fubini's theorem, since $\alpha(u)$ is a nondecreasing bounded function. Thus the proof is complete.

Lemma 5. A necessary and sufficient condition that

$$
u(x, t)=\int_{0}^{\infty} e^{-t u^{2}} g(x u) d \alpha(u), \quad t>0
$$

with $\alpha(u)$ nondecreasing and bounded is that

(i) $\Delta_{x} u(x, t)=-(\partial / \partial t) u(x, t), t>0$,

(ii) $u(z, t)$ is analytic for each $t>0$ and $|\operatorname{Re} z|<R$,

(iii) $u(i x, t)>-M$ for some $M>0, t>0$,

(iv) $u(i x, 0+)$ exists and is positive definite.

Proof. The necessity of the conditions are immediate and the sufficiency follows on noting that $u(x,-t)$ is a generalized temperature for $t<0$, and hence $u(i x, t)$ is a generalized temperature for $t>0$. Thus $u(i x, t)$ satisfies the conditions of Lemma 4 and hence the desired integral representation follows for $u(x, t)$.

Lemmas 4 and 5 yield the following result whose proof is established analogously to that for Theorem 3 and hence will be omitted.

Theorem 6. A function $u(x, y, t)$ has the representation

$$
u(x, y, t)=\int_{0}^{\infty} e^{-t u^{2}} g(x u) g(y u) d \alpha(u), \quad t>0
$$

with $\alpha(u)$ nondecreasing and bounded iff 
(i) $\Delta_{x} u(x, y, t)=-\Delta_{y} u(x, y, t)=(\partial / \partial t) u(x, y, t), t>0$.

(ii) For $x \geqq 0, t>0, u(x, z, t)$ is analytic for $|\operatorname{Re} z|<R$,

(iii) $u(x, i y, t)>-M$,

(iv) $u(x, 0,0+), u(0, i y, 0+)$ exist and are positive definite.

The theorem is illustrated by the function

$$
u(x, y, t)=e^{-t a^{2}} g(x a) g(y a)
$$

which satisfies the conditions of the theorem and which has the representation

$$
u(x, y, t)=\int_{0}^{\infty} e^{-t u^{2}} g(x u) g(y u) d \alpha(u)
$$

where $\alpha(u)$ is constant except for a unit positive jump at $u=a$.

\section{REFERENCES}

1. F. M. Cholewinski, D. T. Haimo and A. E. Nussbaum, $A$ necessary and sufficient condition for the representation of a function as a Hankel-Stieltjes transform, Studia Math. 37 (1970), 57-62.

2. F. M. Cholewinski and D. T. Haimo, The Weierstrass-Hankel convolution transform, J. Analyse Math. 17 (1966), 1-58. MR 35 \#5866.

3. - Integral representations of solutions of the generalized heat equation, Illinois J. Math. 10 (1966), 623-638. MR 33 \#7796.

4. D. T. Haimo, Expansions in terms of generalized heat polynomials and of their Appell transforms, J. Math. Mech. 15 (1966), 735-758. MR 33 \#4340.

5. - Generalized temperature functions, Duke Math. J. 33 (1966), 305-322. MR 34 \#1802.

6. - Series representation of generalized temperature functions, SIAM J. Appl. Math. 15 (1967), 359-367. MR 35 \#3204.

7. - Equivalence of integral transform and series expansion representation of generalized temperatures, Analytic Methods in Mathematical Physics, Gordon and Breach, New York, 1970, pp. 453-4.59.

8. D. V. Widder, Functions of three variables which satisfy both the heat equation and Laplace's equation in two variables, J. Austral. Math. Soc. 3 (1963), 396-407. MR $28 \# 4140$.

9. ——, A problem of Kampe de Feriet, J. Math. Anal. Appl. 9 (1964), 458-467. MR 34 \#8107.

University of Missouri-St. Louis, St. Louis, Missouri 63121 